\title{
The important herbal pair for the treatment of COVID-19 and its possible mechanisms
}

\author{
Shujie Xia', Zhangfeng Zhong², Bizhen Gao ${ }^{1}$, Chi Teng Vong ${ }^{2}$, Xuejuan Lin', Jin Cai ${ }^{3}$, Hanlu Gao ${ }^{2}$, Ging Chan² \\ and Candong $\mathrm{Li}^{1^{*}}$ (i)
}

\begin{abstract}
Background: Coronavirus Disease 2019 (COVID-19) is an unprecedented disaster for people around the world. Many studies have shown that traditional Chinese medicine (TCM) are effective in treating COVID-19. However, it is difficult to find the most effective combination herbal pair among numerous herbs, as well as identifying its potential mechanisms. Herbal pair is the main form of a combination of TCM herbs, which is widely used for the treatment of diseases. It can also help us to better understand the compatibility of TCM prescriptions, thus improving the curative effects. The purpose of this article is to explore the compatibility of TCM prescriptions and identify the most important herbal pair for the treatment of COVID-19, and then analyze the active components and potential mechanisms of this herbal pair.

Methods: We first systematically sorted the TCM prescriptions recommended by the leading experts for treating COVID-19, and the specific herbs contained in these prescriptions across different stages of the disease. Next, the association rule approach was employed to examine the distribution and compatibility among these TCM prescriptions, and then identify the most important herbal pair. On this basis, we further investigated the active ingredients and potential targets in the selected herbal pair by a network pharmacology approach, and analyzed the potential mechanisms against COVID-19. Finally, the main active compounds in the herbal pair were selected for molecular docking with severe acute respiratory syndrome coronavirus 2 (SARS-COV-2) 3CLpro and angiotensin converting enzyme II (ACE2) for further verification.
\end{abstract}

Result: We obtained 32 association rules for the herbal combinations in the selection of TCM treatment for COVID-19. The results showed that the combination of Amygdalus Communis Vas (ACV) and Ephedra sinica Stapf (ESS) had the highest confidence degree and lift value, as well as high support degree, which can be used in almost all the stages of COVID-19, so ACV and ESS (AE) were selected as the most important herbal pair. There were 26 active ingredients and 44 potential targets, which might be related to the herbal pair of AE against COVID-19. The main active ingredients of AE against COVID-19 were quercetin, kaempferol, luteolin, while the potential targets were Interleukin 6 (IL-6), Mitogen-activated Protein Kinase 1 (MAPK)1, MAPK8, Interleukin-1 $\beta$ (IL-1 $\beta$ ), and Nuclear factor kappa-light-chain-enhancer of activated B cells (NF-kB) p65 subunit (RELA). The protein-protein interaction (PPI) cluster demonstrated that IL-6 was the seed in the cluster, which plays an important role in connecting other nodes in the PPI network. The potential

\footnotetext{
*Correspondence: gchan@um.edu.mo; fizylcd@126.com

${ }^{1}$ Research Base of Traditional Chinese Medicine Syndrome, Fujian

University of Traditional Chinese Medicine, No.1 Qiuyang Road, Minhou District, 350122 Fuzhou, China

2 State Key Laboratory of Quality Research in Chinese Medicine, Institute of Chinese Medical Sciences, University of Macau, 999078 Macao, China

Full list of author information is available at the end of the article
}

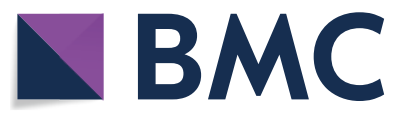

(c) The Author(s) 2021. This article is licensed under a Creative Commons Attribution 4.0 International License, which permits use, sharing, adaptation, distribution and reproduction in any medium or format, as long as you give appropriate credit to the original author(s) and the source, provide a link to the Creative Commons licence, and indicate if changes were made. The images or other third party material in this article are included in the article's Creative Commons licence, unless indicated otherwise in a credit line to the material. If material is not included in the article's Creative Commons licence and your intended use is not permitted by statutory regulation or exceeds the permitted use, you will need to obtain permission directly from the copyright holder. To view a copy of this licence, visit http://creativeco mmons.org/licenses/by/4.0/. The Creative Commons Public Domain Dedication waiver (http://creativecommons.org/publicdomain/ zero/1.0/) applies to the data made available in this article, unless otherwise stated in a credit line to the data. 
pathways mainly involved tumor necrosis factor (TNF), Toll-like receptor (TLR), hypoxia-inducible factor-1 (HIF-1), and nucleotide-binding oligomerization domain (NOD)-like receptor (NLRs). The molecular docking results showed that the main active ingredients of AE have good affinity with SARS-COV-2 3CLpro and ACE2, which are consistent with the above analysis.

Conclusions: There were 32 association rules in the TCM prescriptions recommended by experts for COVID-19. The combination of ACV and EAS was the most important herbal pair for the treatment of COVID-19. AE might have therapeutic effects against COVID-19 by affecting the inflammatory and immune responses, cell apoptosis, hypoxia damage and other pathological processes through multiple components, targets and pathways.

Keywords: Novel coronavirus pneumonia, Association rules, Ephedra sinica Stapf, Amygdalus Communis Vas, Network pharmacology, Herbal pair, Molecular docking

\section{Background}

Coronavirus Disease 2019 (COVID-19), that was discovered in December 2019, is an unprecedented disaster for people around the world. This disease is caused by a novel coronavirus (SARS-CoV-2). It is found that SARS$\mathrm{CoV}-2$ invades cells by binding its spinous $\mathrm{S}$ protein with angiotensin converting enzyme II (ACE2) receptor on the surface of human cells. In clinical, the characteristics of COVID-19 are strong infectivity, rapid development and general susceptibilities [1]. Patients with COVID19 showed typical respiratory symptoms (such as fever, cough and lung damage) and some other symptoms, such as fatigue, myalgia, and diarrhea [2]. Many studies have found that asymptomatic people also have strong infectivity $[3,4]$. At present, the main treatment strategy for COVID-19 is supportive care, which is supplemented by the combination of broad-spectrum antibiotics, antivirals, corticosteroids and convalescent plasma [5].

In China, it has become a consensus to combine traditional Chinese and Western medicine to improve the curative effect of a disease and reduce the mortality rate. The facts have shown that traditional Chinese medicine (TCM) always plays an important role in the prevention and treatment of infectious diseases such as Severe Acute Respiratory Syndrome (SARS) in 2003 and influenza A (H1N1) in 2009 [6]. Several studies have found that integrated medicine has better effects in improving the cure rate and overall response rate, and does not increase adverse drug reactions for COVID-19 [7]. Until now, seven consecutive versions of "diagnosis and treatment protocol for COVID-19" have been issued by the National Health Commission of the People's Republic of China, which are mainly based on the characteristics of different stages of the disease and the symptoms of patients [8]. Many studies have shown that TCM, such as Lianhua Qingwen capsule, Qingfei Paidu decoction, are indeed effective in treating COVID-19 by inhibiting virus replication and invasion or inflammatory responses $[9$, $10]$.
The key to obtain satisfactory curative effect is divided into two points [11], the first point is to get accurate syndrome differentiation, and the second point is to identify reasonable compatibility of traditional medicine. In the selection of TCM, identifying the compatibility of herbal combination among different herbs are of great significance to improve the effectiveness of the treatment. Herbal pair is the basic form of the compatibility of traditional medicine, which consists of two kinds of herbs, and they exert therapeutic effects in a synergistic manner. The study of herbal pairs for the treatment of COVID-19 can help to understand the compatibility of TCM prescriptions, thus improving the curative effects. Therefore in this study, we used the most frequent herbal pair as the most important herbs for the treatment of COVID-19. In this way, we can better grasp the focus of treatment using TCM and further explore the underlying mechanisms of exerting therapeutic effects against COVID-19. However, due to a number of TCM prescriptions involved in the treatment of COVID-19, it is difficult to find the effective combination compatibility and the most commonly used herbal pair among numerous herbs, especially for those inexperienced doctors. In addition, further research on the active ingredients, potential targets and the mechanisms of action of Chinese medicine are crucial to the precise treatment of TCM for COVID-19.

Therefore, the aim of this study was to utilize the association rule approach to examine the distribution and combination compatibility among TCM recommended by leading experts for the treatment of COVID-19, and then identify the most important herbal pair. The association rule approach has been used in many studies to explore the law of TCM combination. For example, You et al. used Apriori algorithm to explore the medication rules of kidney-tonifying method in treating bone marrow suppression and found 26 herb suits association rules [12]. After screening out the most important herbal pair through association rules, we further investigated the active ingredients and potential targets of the selected 
herbal pair by a network pharmacology approach, a systematic method proposed by Shao Li from Tsinghua University, China [13]. In this study, by applying the association rules, we could obtain many interesting rules to investigate the hidden relationships among numerous TCM prescriptions and select the most important herbal pair. With network pharmacology approach, we could further analyze the effective components, potential targets and underlying mechanisms of the most important herbal pair. At last, we connect the main active components of AE with SARS-CoV-2 and ACE2 by molecular docking, so as to verify the above analysis and provide some basis for its further experimental research and clinical application.

\section{Methods}

\section{Compatibility of TCM}

According to the Chinese medicine prevention and treatment protocol formulated by domestic first-line experts in the diagnosis and treatment protocol for COVID-19 [9], the TCM prescriptions are recommended according to different disease stages that include observation period, mild, middle, severe and recovery stages. On this basis, each herb in these prescriptions was classified and summarized to identify the most commonly used TCM for the treatment of COVID-19. Then, the data mining method of the association rules was used to mine the combination rule of TCM and find the important herbal pairs for the treatment of COVID-19. Association rule analysis is an important method to reveal the internal structural characteristics of the data, i.e. mining the correlation between different variables if a proper support and confidence are given [14]. This can be done by the Apriori algorithm provided from the 'arules' package of the $\mathrm{R}$ software.

The Apriori algorithm was used as follows: Firstly, it was used to determine all the frequent sets that are satisfied with a minimum support degree and minimum confidence degree. Then, it was used to generate strong association rules from these frequent item sets. In this study, we set the support degree to 0.2 and the confidence degree to 0.6. The data format was sorted into the "shopping basket" format. The herbs included in each of the prescriptions were analyzed to observe the "co-occurrence" of different herbs, and the frequent items of the data, and the combination rules of herbal pairs were also analyzed. Finally, the most important herbal pair for the treatment of COVID-19 was selected for further network pharmacological analysis.

\section{Active ingredients and targets of the selected herbal pair} The active ingredients of the selected herbal pair were obtained from the Traditional Chinese Medicine
Systems Pharmacology Database (TCMSP, https://tcmsp w.com/tcmsp.php/) and PubChem Database (https:// pubchem.ncbi.nlm.nih.gov/). TCMSP is a systematic pharmacology platform designed for herbs, which is capable of describing the relationship between drugs, targets and diseases [15]. PubChem is the world's largest collection of accessible chemical information, which can provide the molecular formula, structure, biological activities and toxicity information of the chemical compounds. In addition, the TCMSP database was used to identify potential targets of the herbal pair, and their gene names were obtained from the UniProt database (https://www.uniprot.org/) by limiting the species with "Homo sapiens".

In this section, the active ingredients were extracted based on the pharmacokinetic evaluation (absorption, distribution, metabolism, excretion (ADME) properties of the compounds) to identify the chemical ingredients with favorable pharmacokinetics properties [16]. Therefore, we employed two important ADME-related properties, namely, oral bio-availability $(\mathrm{OB})$ and druglikeness (DL), in our study to explore the potential bioactive compounds of $\mathrm{AE}$. The ingredients with $\mathrm{OB} \geq 30 \%$ and $\mathrm{DL} \geq 0.18$ were selected in this study, and all of the candidate compounds were approved through literature reviews.

\section{Potential targets of the herbal pair for COVID-19}

The data for the COVID-19-associated target genes were obtained from Genecards database (https://www.genec ards.org/) and the Online Mendelian Inheritance in Man (OMIM) database (https://omim.org/). Genecards is an extensive platform which provides insight into predicted and annotated human genes. All of the gene-centric data were gathered from 150 web resources, including genetic, genomic, transcriptomic, proteomic and functional information [17]. The OMIM database links and classifies all the known diseases with a genetic component, and provides information to the genomic analyses of catalogued genes [18]. The keyword was set as "novel coronavirus pneumonia".

The mapping of COVID-19 disease and drug targets was carried out by the $R$ (3.6.2) software to identify the intersection targets as potential targets. Then, we obtained the protein-protein interaction (PPI) data of intersection targets from the STRING database (https:// string-db.org/). By choosing the "multiple proteins" mode and setting the protein species as "homo sapiens". The STRING database defines PPI with confidence ranges for data scores (high $>0.7$; medium $>0.4$; low $>0.15$ ) [19]. In this study, we selected a confidence score of $>0.7$ to construct our PPI network. Next, we imported the .tsv file into Cytoscape for further analysis. 
Construction of "herb-component-target-disease" network Network analysis was conducted to facilitate scientific interpretation of the complicated relationships among herbs, compounds, diseases and genes [20]. In this study, we generated the networks using Cytoscape (version 3.7.2). Firstly, the active components that are corresponding to the targets of COVID-19 were identified by the $R$ software, and the disease-related active components and potential targets were imported into the Cytospace software to further construct the "herb-component-targetdisease" network. The centrality of the network nodes was analyzed by CytoNCA. The degree value was used as the screening condition. The higher the degree value, the more targets the component was related to. In this way, we could analyze the disease-related core components of the $\mathrm{AE}$ herbal pair.

\section{Enrichment analysis}

In this study, the background database "org. HS. Eg.db" of R3.6.2 was used to obtain the gene ID (Entrez ID) of the potential targets, and then the "clusterprofiler" package was used to analyze the Gene Ontology (GO) function enrichment of these potential targets, including three aspects: biological process (BP), cellular component (CC) and molecular function (MF). The $p$-value was set with a cutoff of 0.05 and $q$-value with a cutoff of 0.05 , and each category was ranked by significancy. The top 10 enrichment items were displayed in form of a histogram. Kyoto Encyclopedia of Genes and Genomes (KEGG) analysis was used to obtained potential targets by the DAVID database. The $\mathrm{R}$ software was used to analyze the gene number, significancy, enrichment fold of the target-related pathways by drawing a bubble diagram. Then, we imported KEGG data into Cytoscape to conduct network analysis of the top 20 enrichment pathways with significant differences and their associated potential targets.

\section{Autodock method}

The top five ranked compounds selected by the number of targets in ESS and ACV, were used to dock with SARSCoV-2 3CLpro and ACE2, respectively. Firstly, the threedimensional structures of 10 active compounds were obtained from Pubchem database (https://pubchem.ncbi. nlm.nih.gov/). The protein structure of SARS-CoV-2 3CLpro (6LU7) and ACE2 (1R42) were were downloaded from PDB database (www.rcsb.org/). And then, Pymol software was used to remove the small molecule ligands and water molecules for the receptor proteins. Finally, Aotudock vina software was used for molecular docking and the results of binding energy repeated three times. Here, Binding energy less than $-6.0 \mathrm{~kJ} / \mathrm{mol}$ was used as the screening standard.

\section{Results}

Compatibility law of Chinese medicine for COVID-19

We collected a total of 24 TCM prescriptions including 105 herbs that are highlighted in the guidelines for the treatment of COVID-19 [9]. According to the characteristics of the disease progression, we summarized TCM prescriptions that are available at different stages of the disease and their specific herbs in Fig. 1. In general, it was found that different TCM prescriptions are recommended at different stages of COVID-19. Even at the same stage, the recommended TCM prescriptions vary according to the specific syndromes of the patients. Among these prescriptions, Qingfei Paidu Decoction is the most recommended prescription, which can be used for mild, moderate and severe patients with COVID19. Besides, Xuebijing Injection, Reduning Injection, Tanreqing Injection and Xingnaojing Injection were recommended twice, in severe and critical conditions. Moreover, it appeared that almost every prescription consists of a variety of different herbs, and the same herb may appear in different prescriptions by certain combination compatibility.

To further explore the prescription rules of TCM treatment for COVID-19, we analyzed 105 kinds of herbs in the recommended TCM prescriptions, and found that the most commonly used herbs were Licorice, Gypsum, Ephedra sinica Stapf, Agastache rugosa, Amygdalus Communis Vas, Baikal Skullcap, and Forsythia suspensa. The top 20 commonly used Chinese herbs were shown in Fig. 2a. In order to generate association rules among these herbs, we performed data analysis based on the association rules algorithm in 24 sets of recommended TCM prescriptions. We obtained 32 association rules for herbal combinations from the TCM prescriptions of COVID-19 (Table 1). In general, a big value for the lift $(\mathrm{X} \Rightarrow \mathrm{Y})$ indicates a stronger association between $\mathrm{X}$ and $Y$ [21]. The lift values of these 32 rules were all greater than 1 , this indicated that there were positive interdependence effects on these rules.

\section{Screening of important herbal pairs}

We further extracted the combination herb pairs containing two herbs by subset function. There were 16 rules for the combination compatibility of herbal pairs. According to the confidence ranking, Amygdalus Communis Vas $(\mathrm{ACV})\}=>\{$ Ephedra sinica Stapf (ESS) $\}, \quad\{$ Poria $\operatorname{cocos}\}=>\{$ Agastache rugosa $\}$, $\{\mathrm{ACV}\}=>\{$ Gypsum $\},\{$ Gypsum $\} \Rightarrow>\{$ Licorice $\},\{$ ESS $\}$ $=>\{A C V\}$ and $\{E S S\}=>\{$ Gypsum $\}$ were the top six important herbal pairs, which were shown in Fig. 2 b. The larger the circle between the two herbs, the higher the confidence level. The darker the color, the higher the lift value. Among them, $\{\mathrm{ACV}\}=>\{\mathrm{ESS}\}$ has the 


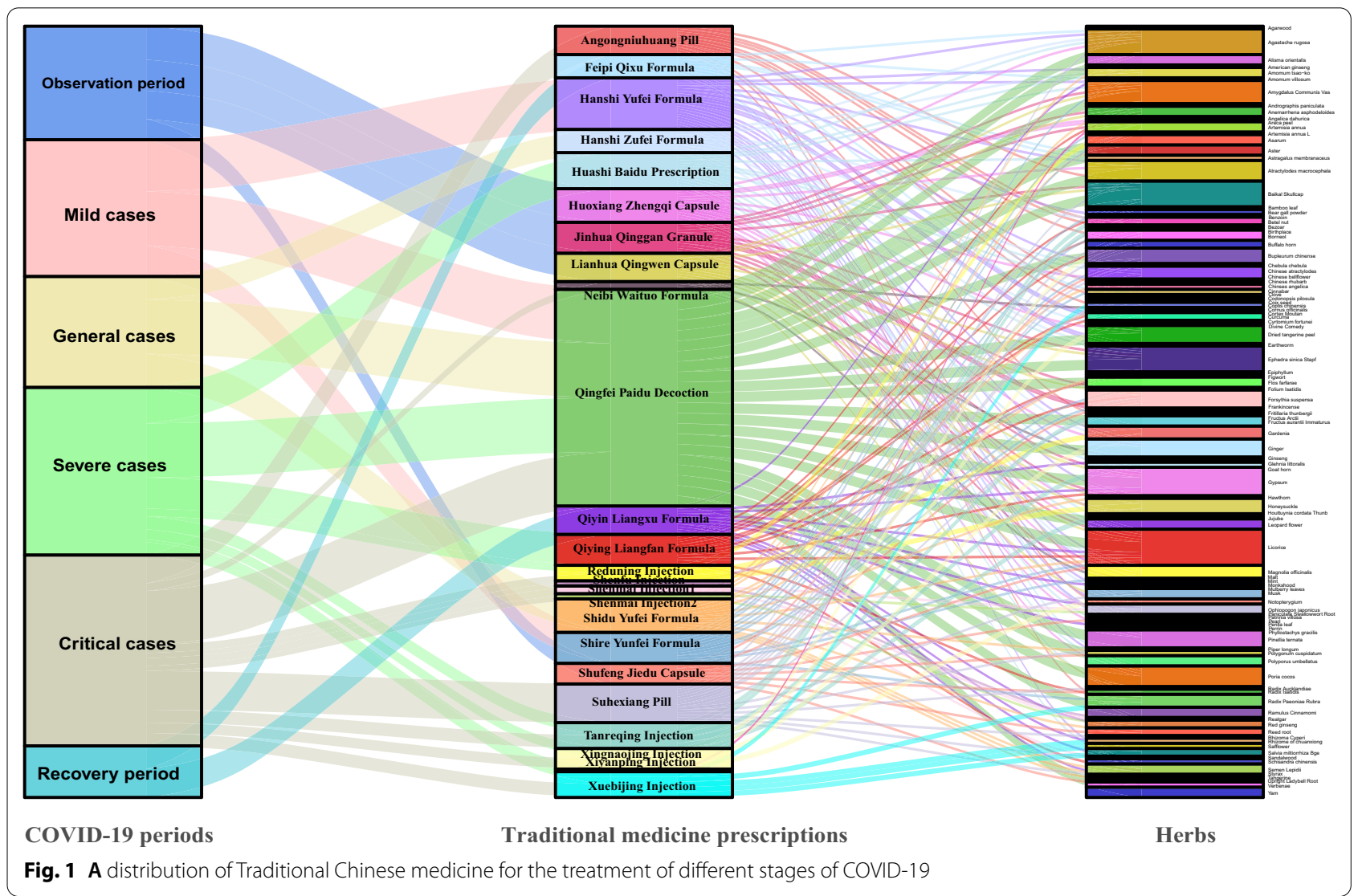

highest confidence degree (1.00) and lift value (3.43), as well as high support degree (0.25). This suggested that the probability of using both herbs at the same time is $25 \%$. Under the premise of using ACV, the probability of using ESS is $100 \%$, which is 3.43 times higher than that of using ESS alone. In addition, further analysis showed that the herbal pair of ACV and ESS was widely used in almost all stages of the disease, including observation period, mild, general, severe and critical conditions. Therefore, the combination of ACV and ESS was selected as the most important herbal pair for the treatment of COVID-19.

\section{The active ingredients of ACV and ESS (AE)}

Based on the above analysis, the combination of ACV and ESS was regarded as the most important herbal pair, and its active ingredients and potential targets were further studied. 40 active ingredients from 476 compounds of $\mathrm{AE}$ met the requirements of $\mathrm{OB} \geq 30 \%$ and $\mathrm{DL} \geq 0.18$. It was found that 5 active components had no corresponding action targets, and the other
35 active ingredients that could be found by literature reviews had a total of 210 targets (Table 2). Among these 35 active ingredients, 21 ingredients were from ESS, 16 ingredients were from ACV, and 2 ingredients $((+)$-catechin, stigmasterol) were from both herbs.

\section{"AE-component-target-COVID" network}

A total of 261 COVID-19 targets were obtained from GeneCards and OMIM database, and 44 crossed targets of AE and COVID-19 were obtained by the R software, as shown in Fig. 3a. These crossed targets were regarded as the potential targets of $\mathrm{AE}$ against COVID19. Then, the "AE-component-target-disease" network was constructed by Cytoscape, as depicted in Fig. 3c. This network included 73 nodes and 225 edges, with a network density of 0.081 and a network diameter of 4 . The key nodes in this network were shown in Table 3. The topological parameters showed that the nodedegree distribution obeyed the power-law distribution (Fig. 3b). There were 43 ESS targets, 7 ACV targets and 6 overlapped targets (PTGS2, PTGS1, CAT, NOS2, PPARG, and SOD1) from a total of 26 active ingredients 


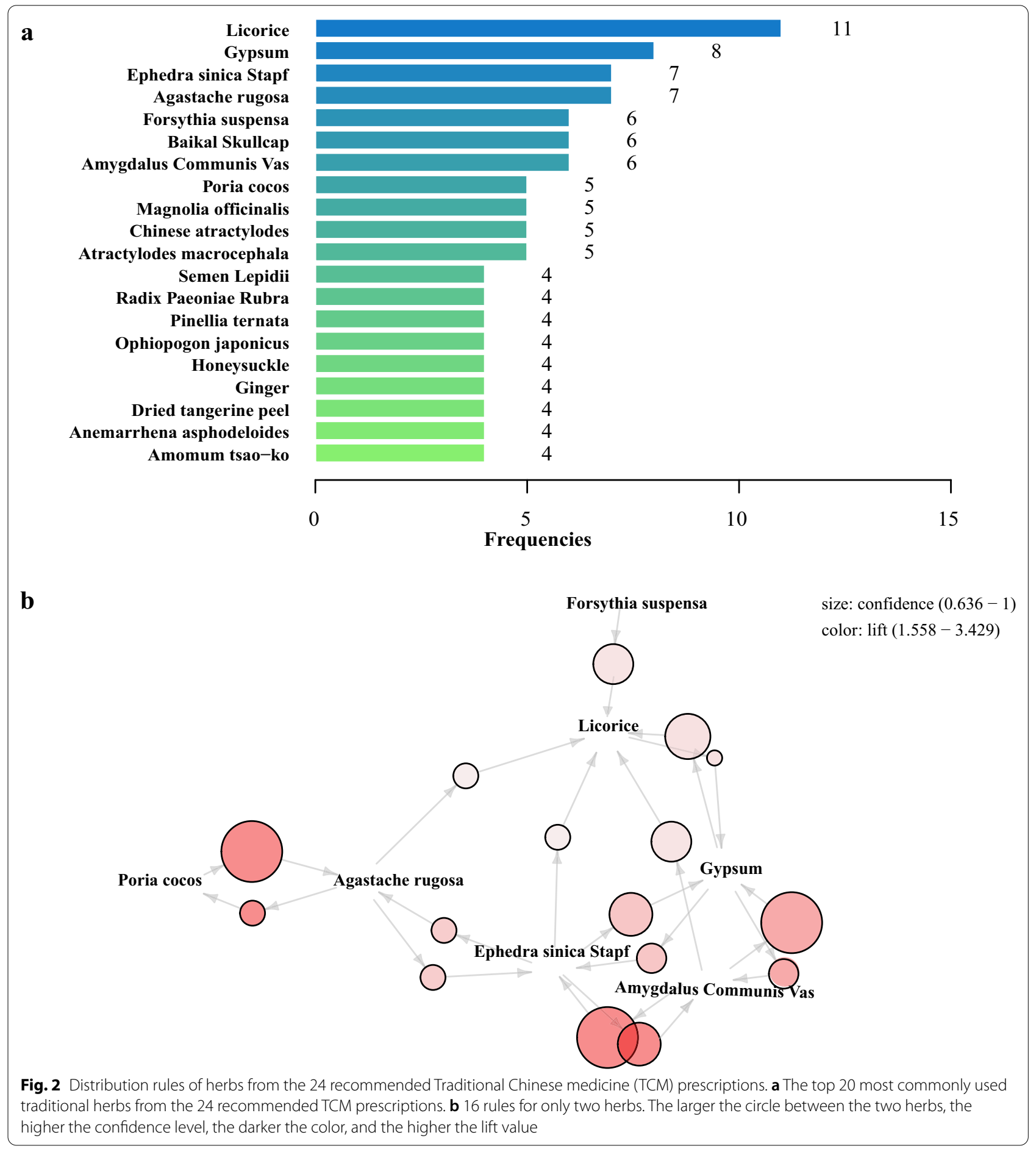

of AE. According to the degree value, the most critical ingredients of $\mathrm{AE}$ were quercetin, luteolin, kaempferol, naringenin and (+)-catechin, which interacted with 38 , $18,13,10$ and 7 targets of COVID-19, respectively.

\section{PPI network of AE against COVID-19}

PPI network has been widely used to identify many different interactions of the protein targets in the context of a complex disease. There were a total of 44 nodes and 324 interaction lines in the STRING PPI network. 
Table 1 Meaningful association rules among Traditional Chinese herbs for the treatment of COVID-19

\begin{tabular}{|c|c|c|c|}
\hline Rules & Confidence & Support & Lift \\
\hline$\{$ Amygdalus Communis Vas $\}=>$ Ephedra sinica Stapf $\}$ & 1.000 & 0.250 & 3.429 \\
\hline$\{$ Poria $\operatorname{cocos}\}=>\{$ Agastache rugosa $\}$ & 1.000 & 0.208 & 3.429 \\
\hline$\{$ Amygdalus Communis Vas $\}=>\{$ Gypsum $\}$ & 1.000 & 0.250 & 3.000 \\
\hline$\{$ Ephedra sinica Stapf,Amygdalus Communis Vas $\}=>\{$ Gypsum $\}$ & 1.000 & 0.250 & 3.000 \\
\hline$\{$ Gypsum,Amygdalus Communis Vas $\}=>\{$ Ephedra sinica Stapf $\}$ & 1.000 & 0.250 & 3.429 \\
\hline$\{$ Ephedra sinica Stapf,Gypsum $\}=>$ Amygdalus Communis Vas $\}$ & 1.000 & 0.250 & 4.000 \\
\hline$\{$ Licorice,Amygdalus Communis Vas $\}=>\{$ Ephedra sinica Stapf $\}$ & 1.000 & 0.208 & 3.429 \\
\hline$\{$ Licorice,Ephedra sinica Stapf $\}=>\{$ Amygdalus Communis Vas $\}$ & 1.000 & 0.208 & 4.000 \\
\hline$\{$ Licorice,Amygdalus Communis Vas $\}=>\{$ Gypsum $\}$ & 1.000 & 0.208 & 3.000 \\
\hline$\{$ Licorice,Ephedra sinica Stapf $\}=>\{$ Gypsum $\}$ & 1.000 & 0.208 & 3.000 \\
\hline$\{$ Licorice,Ephedra sinica Stapf,Amygdalus Communis Vas $\}=>\{$ Gypsum $\}$ & 1.000 & 0.208 & 3.000 \\
\hline$\{$ Licorice,Gypsum,Amygdalus Communis Vas $\}=>\{$ Ephedra sinica Stapf $\}$ & 1.000 & 0.208 & 3.429 \\
\hline$\{$ Licorice,Ephedra sinica Stapf,Gypsum $\}=>\{$ Amygdalus Communis Vas $\}$ & 1.000 & 0.208 & 4.000 \\
\hline$\{$ Gypsum $\}=>\{$ Licorice $\}$ & 0.875 & 0.292 & 1.909 \\
\hline$\{$ Ephedra sinica Stapf $\}=>$ Amygdalus Communis Vas $\}$ & 0.857 & 0.250 & 3.429 \\
\hline$\{$ Ephedra sinica Stapf $\}=>\{$ Gypsum $\}$ & 0.857 & 0.250 & 2.571 \\
\hline$\{$ Forsythia suspensa $\}=>\{$ Licorice $\}$ & 0.833 & 0.208 & 1.818 \\
\hline$\{$ Amygdalus Communis Vas $\}=>\{$ Licorice $\}$ & 0.833 & 0.208 & 1.818 \\
\hline$\{$ Ephedra sinica Stapf,Amygdalus Communis Vas $\}=>\{$ Licorice $\}$ & 0.833 & 0.208 & 1.818 \\
\hline$\{$ Gypsum,Amygdalus Communis Vas $\}=>\{$ Licorice $\}$ & 0.833 & 0.208 & 1.818 \\
\hline$\{$ Ephedra sinica Stapf,Gypsum $\}=>\{$ Licorice $\}$ & 0.833 & 0.208 & 1.818 \\
\hline$\{$ Ephedra sinica Stapf,Gypsum,Amygdalus Communis Vas $\}=>\{$ Licorice $\}$ & 0.833 & 0.208 & 1.818 \\
\hline$\{$ Gypsum $\}=>\{$ Amygdalus Communis Vas $\}$ & 0.750 & 0.250 & 3.000 \\
\hline$\{$ Gypsum $\}=>\{$ Ephedra sinica Stapf $\}$ & 0.750 & 0.250 & 2.571 \\
\hline$\{$ Agastache rugosa $\}=>\{$ Poria cocos $\}$ & 0.714 & 0.208 & 3.429 \\
\hline$\{$ Agastache rugosa $\}=>\{$ Ephedra sinica Stapf $\}$ & 0.714 & 0.208 & 2.449 \\
\hline$\{$ Ephedra sinica Stapf $\}=>\{$ Agastache rugosa $\}$ & 0.714 & 0.208 & 2.449 \\
\hline$\{$ Agastache rugosa $\}=>\{$ Licorice $\}$ & 0.714 & 0.208 & 1.558 \\
\hline$\{$ Ephedra sinica Stapf $\}=>\{$ Licorice $\}$ & 0.714 & 0.208 & 1.558 \\
\hline$\{$ Licorice,Gypsum $\}=>$ Amygdalus Communis Vas $\}$ & 0.714 & 0.208 & 2.857 \\
\hline$\{$ Licorice,Gypsum $\}=>\{$ Ephedra sinica Stapf $\}$ & 0.714 & 0.208 & 2.449 \\
\hline$\{$ Licorice $\}=>\{$ Gypsum $\}$ & 0.636 & 0.292 & 1.909 \\
\hline
\end{tabular}

The PPI enrichment $p$-value was less than $1.0 \mathrm{e}-16$, demonstrating an obvious protein interaction relationship, which was shown in Fig. 4a. Due to the complexity of the original network obtained from the STRING database, we imported the PPI data into Cytoscape to explore the importance of potential targets in the protein networks and the main cluster in this network. The node represents the potential targets, and the larger the node area and the redder the color, the more important the target protein is. As shown in Fig. 4b, interleukin 6 (IL-6), mitogen-activated protein kinase (MAPK) 1, MAPK8, interleukin-1 $\beta$ (IL-1 $\beta$ ), nuclear factor kappalight-chain-enhancer of activated $B$ cells (NF-kB) p65 subunit (RELA), C-X-C motif chemokine ligand 8 (CXCL-8), C-C motif chemokine ligand 2 (CCL2) and prostaglandin $\mathrm{G} / \mathrm{H}$ synthase 2 (PTGS2) were the key target proteins for the treatment of COVID-19 with AE. Among them, IL-6 (degree = 32) was the most critical target in the PPI network, and the main cluster network of IL-6 was shown in Fig. 4c.

\section{Enrichment of potential targets of $\mathrm{AE}$}

To further explore the underlying mechanisms of AE as a therapy against COVID-19, we performed GO enrichment and KEGG analysis with the 44 potential targets identified by the $\mathrm{R}$ software. GO enrichment consists of three parts, biological process (BP), cellular component (CC) and molecular function (MF). There were $1633 \mathrm{GO}$ enrichment terms for BP, and the most enriched terms included responses to lipopolysaccharide (LPS), bacterial 
Table 2 The active ingredients of ACV and ESS

\begin{tabular}{|c|c|c|c|c|c|}
\hline Herb & Mol Name & Mol Name & OB\% & $\mathrm{DL}$ & Targets \\
\hline \multirow[t]{21}{*}{ ESS } & MOL000098 & Quercetin & 46.43 & 0.28 & 154 \\
\hline & MOL000422 & Kaempferol & 41.88 & 0.24 & 63 \\
\hline & MOL000006 & Luteolin & 36.16 & 0.25 & 57 \\
\hline & MOL000358 & $\beta$-sitosterol & 36.91 & 0.75 & 38 \\
\hline & MOL004328 & Naringenin & 59.29 & 0.21 & 37 \\
\hline & MOL000449 & Stigmasterol & 43.83 & 0.76 & 31 \\
\hline & MOL002823 & Herbacetin & 36.07 & 0.27 & 14 \\
\hline & MOL005573 & Genkwanin & 37.13 & 0.24 & 14 \\
\hline & MOL004576 & Taxifolin & 57.84 & 0.27 & 12 \\
\hline & MOL005842 & Pectolinarigenin & 41.17 & 0.3 & 12 \\
\hline & MOL000492 & (+)-catechin & 54.83 & 0.24 & 11 \\
\hline & MOL002881 & Diosmetin & 31.14 & 0.27 & 10 \\
\hline & MOL005190 & Eriodictyol & 71.79 & 0.24 & 9 \\
\hline & MOL011319 & Truflex OBP & 43.74 & 0.3 & 7 \\
\hline & MOL010788 & leucopelargonidin & 57.97 & 0.24 & 5 \\
\hline & MOL010489 & Resivit & 30.84 & 0.27 & 4 \\
\hline & MOL007214 & $(+)$-Leucocyanidin & 37.61 & 0.27 & 4 \\
\hline & MOL001494 & Mandenol & 42 & 0.19 & 3 \\
\hline & MOL001755 & 24-Ethylcholest-4-en-3-one & 36.08 & 0.76 & 2 \\
\hline & MOL001771 & poriferast-5-en-3beta-ol & 36.91 & 0.75 & 2 \\
\hline & MOL005043 & campest-5-en-3beta-ol & 37.58 & 0.71 & 1 \\
\hline \multirow[t]{16}{*}{ ACV } & MOL000449 & Stigmasterol & 43.83 & 0.76 & 31 \\
\hline & MOL012922 & I-SPD & 87.35 & 0.54 & 30 \\
\hline & MOL010921 & Estrone & 53.56 & 0.32 & 25 \\
\hline & MOL004908 & Glabridin & 53.25 & 0.47 & 25 \\
\hline & MOL007207 & Machiline & 79.64 & 0.24 & 21 \\
\hline & MOL004841 & Licochalcone B & 76.76 & 0.19 & 19 \\
\hline & MOL005017 & Phaseol & 78.77 & 0.58 & 14 \\
\hline & MOL000492 & $(+)$-catechin & 54.83 & 0.24 & 11 \\
\hline & MOL002311 & Glycyrol & 90.78 & 0.67 & 11 \\
\hline & MOL004903 & Liquiritin & 65.69 & 0.74 & 6 \\
\hline & MOL000953 & CLR & 37.87 & 0.68 & 4 \\
\hline & MOL000359 & Sitosterol & 36.91 & 0.75 & 3 \\
\hline & MOL004355 & Spinasterol & 42.98 & 0.76 & 3 \\
\hline & MOL005030 & gondoic acid & 30.7 & 0.2 & 2 \\
\hline & MOL002211 & 11,14-eicosadienoic acid & 39.99 & 0.2 & 1 \\
\hline & MOL000211 & Mairin & 55.38 & 0.78 & 1 \\
\hline
\end{tabular}

molecules, biotic stimulus and oxidative stress, and positive regulation of cytokine production. Besides, a total of $32 \mathrm{CC}$ items were obtained, and the most enriched terms included membrane raft, microdomain and region, caveola, plasma membrane raft, outer membrane, and focal adhesion. There were $78 \mathrm{GO}$ terms for MF enrichment, and the most enriched terms included cytokine receptor binding and activity, receptor ligand activity, chemokine receptor binding, phosphatase binding, MAP kinase activity, and chemokine activity. The top 10 most important GO items for different categories were shown in Fig. 5.

From KEGG analysis, we obtained a total of 113 pathways that were mainly divided into several categories, such as human diseases, signal transduction, cell process and immune system. The top 20 significantly enriched KEGG pathways were presented in Fig. 6a, including tumor necrosis factor (TNF), Toll-like receptor (TLR), hypoxia-inducible factor-1 (HIF-1), nucleotide-binding oligomerization domain (NOD)-like receptor(NLRs), and several disease-related pathways like Chagas disease and Influenza A. Among these pathways, TNF pathway with relatively lower $p$-value and FDR value $(<0.0001)$ was regarded as an important pathway of $\mathrm{AE}$ against COVID19. The network of the top 20 pathways and their targets was shown in Fig. 6b, where the font size of the label represented the degree value of the node in this network. The detailed information of the gene targets in these 20 pathways was listed in Table 4.

\section{Molecular docking}

The main active compounds in $\mathrm{AE}$, namely Quercetin, Kaempferol, Luteolin, beta-Sitosterol, Naringenin,Stigmasterol, l-SPD, Estrone, Glabridin, Machiline, were used to dock with SARS-COV-2 3CL pro and ACE2 respectively. It is generally believed that the lower the binding energy of ligand and receptor, the more stable the conformation and the greater the possibility of action. The molecular docking results showed that the binding energies of the main active compounds in $\mathrm{AE}$ were all less than $-6 \mathrm{~kJ} / \mathrm{mol}$, which indicates that these compounds can well combine with SARS-COV-2 $3 C L$ pro and ACE2 to play a role in the treatment of COVID-19. The three compounds with the lowest binding energy to SARS-COV-2 3CL pro were Quercetin, Luteoli, Glabridin. The three compounds with the lowest binding energy to ACE2 were Beta-sitosterol, Stigmasterol, Glabridin. The results are shown in Table 5; Fig. 7

\section{Discussion}

Until now, people around the world are still actively fighting against COVID-19, and TCM has played an important role in the treatment of this disease, especially in China. Recent reports from the Information Office of the State Council of China showed that 74,187 patients with COVID-19 received TCM treatment, accounting for $91.5 \%$ of all the cases in China. Even in Hubei province, there were 61,449 patients $(90.6 \%)$ treated by TCM. It has been shown that the total effective rate of TCM treatment for COVID-19 was over $90 \%$. From the above association rule analysis, a total of 32 association rules for herbal combinations was obtained, and these results were consistent with the clinical practice. Among these rules, 


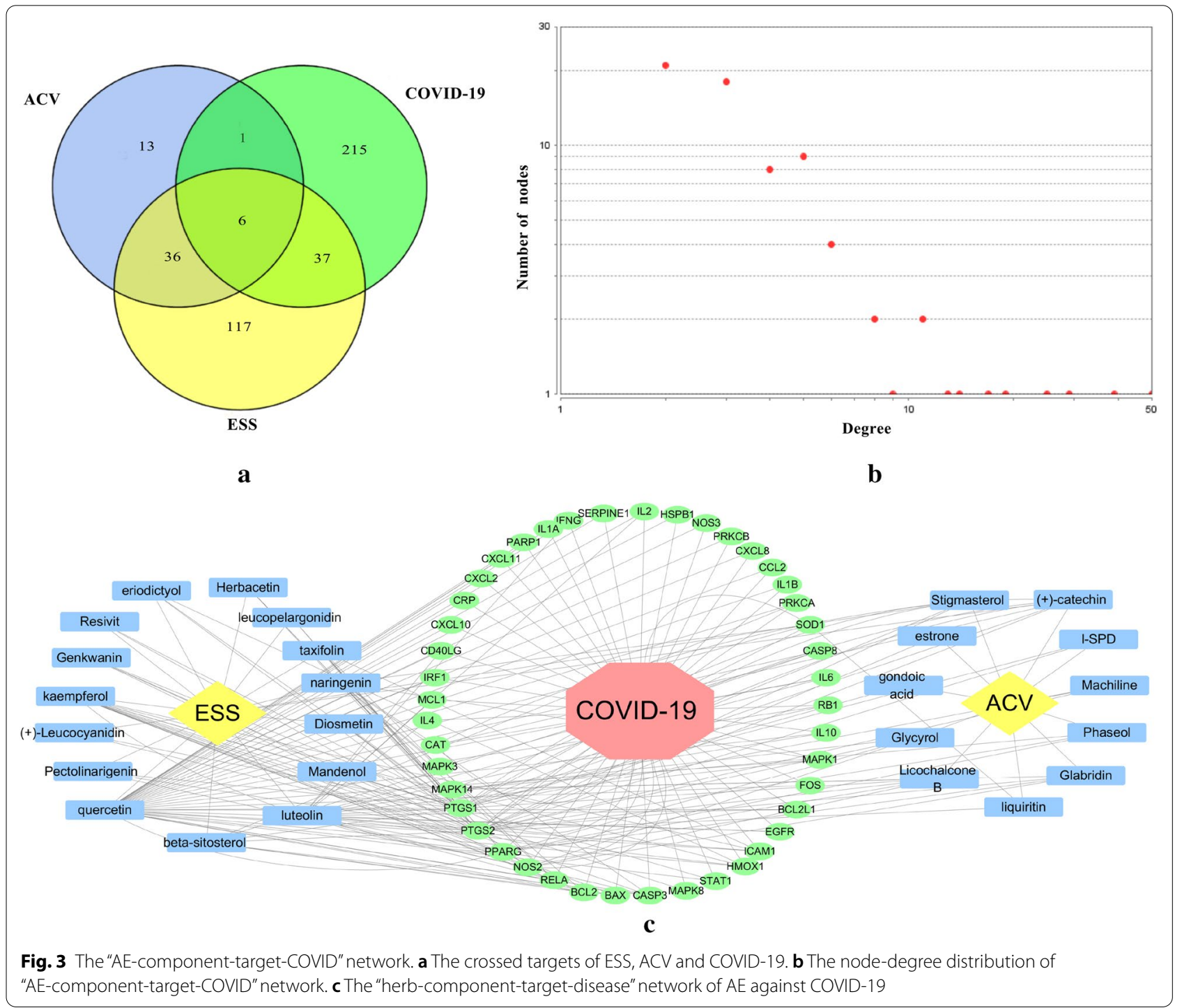

Table 3 Key nodes of "AE-component-target-COVID" network and their topological characteristics

\begin{tabular}{llllll}
\hline Components & $\begin{array}{l}\text { Betweenness Degree Targets } \\
\text { centrality }\end{array}$ & \multicolumn{2}{l}{$\begin{array}{l}\text { Betweenness Degree } \\
\text { centrality }\end{array}$} \\
\hline Quercetin & 0.258 & 39 & PTGS2 & 0.209 & 29 \\
Luteolin & 0.068 & 19 & PTGS1 & 0.147 & 25 \\
Kaempferol & 0.032 & 14 & PPARG & 0.036 & 13 \\
Naringenin & 0.025 & 11 & NOS2 & 0.019 & 9 \\
B-sitosterol & 0.013 & 8 & RELA & 0.007 & 6 \\
(+)-catechin & 0.007 & 8 & CASP3 & 0.006 & 6 \\
Stigmasterol & 0.003 & 6 & SOD1 & 0.009 & 5 \\
Licochalcone B & 0.008 & 6 & MAPK14 & 0.010 & 5 \\
Taxifolin & 0.004 & 5 & ICAM1 & 0.005 & 5 \\
Glycyrol & 0.005 & 5 & HMOX1 & 0.006 & 5 \\
Glabridin & 0.005 & 5 & CAT & 0.003 & 5 \\
\hline
\end{tabular}

the combination of ACV and ESS is one of the most commonly used herbal pair for the treatment of COVID-19, which was not only included in many TCM prescriptions recommended by experts across the country, but it was used throughout the course of COVID-19. Therefore, we suggested that $\mathrm{AE}$ plays an important role in the prevention and treatment of COVID-19. According to the theories of TCM, the main cause of COVID-19 is "dampness toxin", and it mainly affects the function of lung and spleen [22]. Dampness has the characteristics of heavy turbid and sticky that can hinder the movement of Qi and blood, thereby causing lung and spleen dysfunction. ESS has the effects of inducing perspiration, dispelling dampness, diffusing the Lung Qi and relieving cough and asthma, while ACV has the effects of depressing Qi, suppressing cough and relieving asthma. The combination 


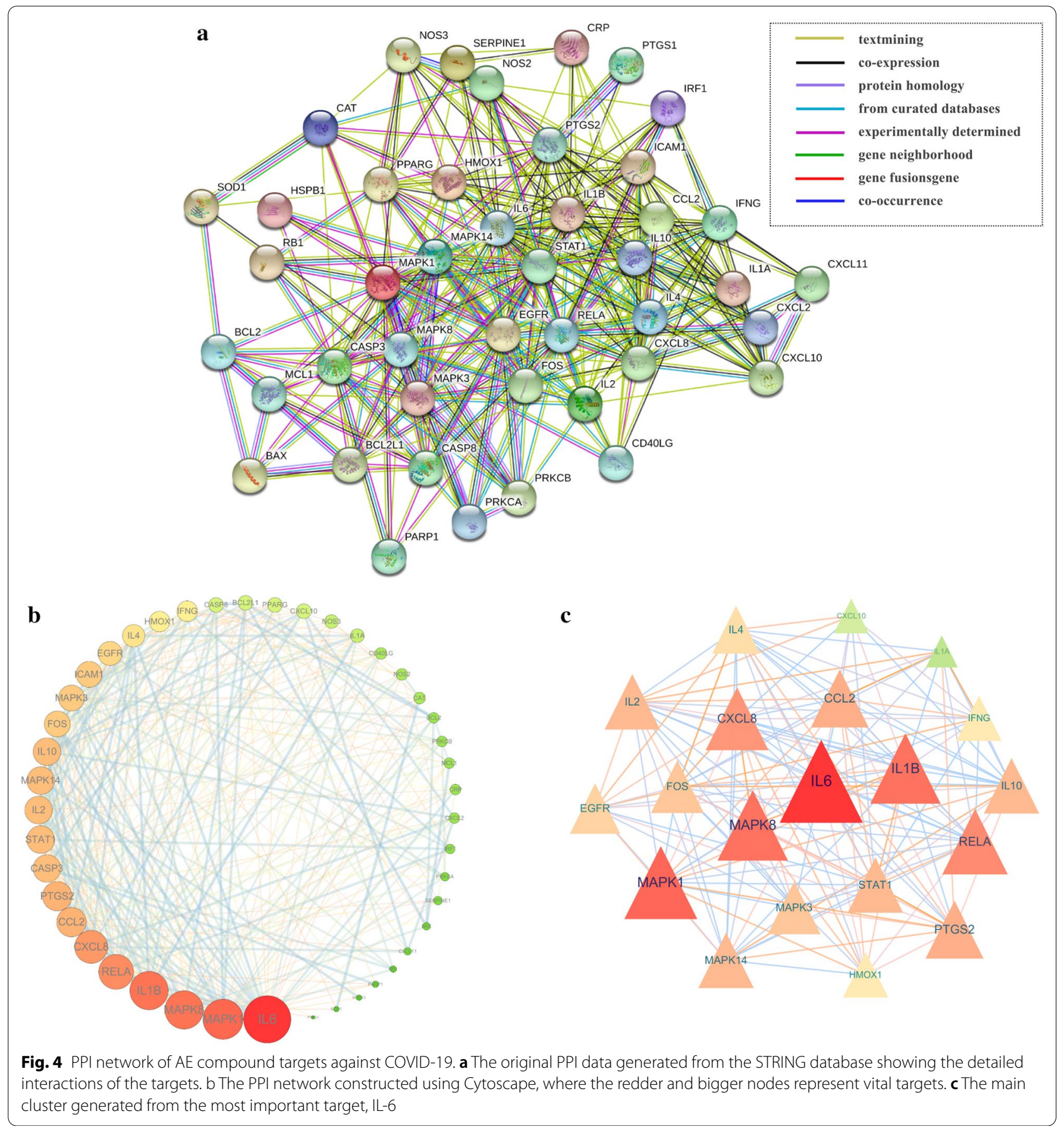

of ESS and ACV can exert the efficacy of normalizing Qi dynamic and relieve cough and asthma. However, its potential mechanisms against COVID-19 have not been fully explored, so we applied network pharmacology approach to further explore the active compounds, potential targets and the underlying mechanisms of $\mathrm{AE}$ against COVID-19.
In this study, we found 26 active ingredients and 44 potential targets, which might be related to AE against COVID-19. After analysis, we found that each herb interacted with an average of 74.5 targets, each compound interacted with an average of 5.73 targets, and each target interacted with an average of $3.48 \mathrm{com}$ pounds. These results indicated that TCM, like ESS and 


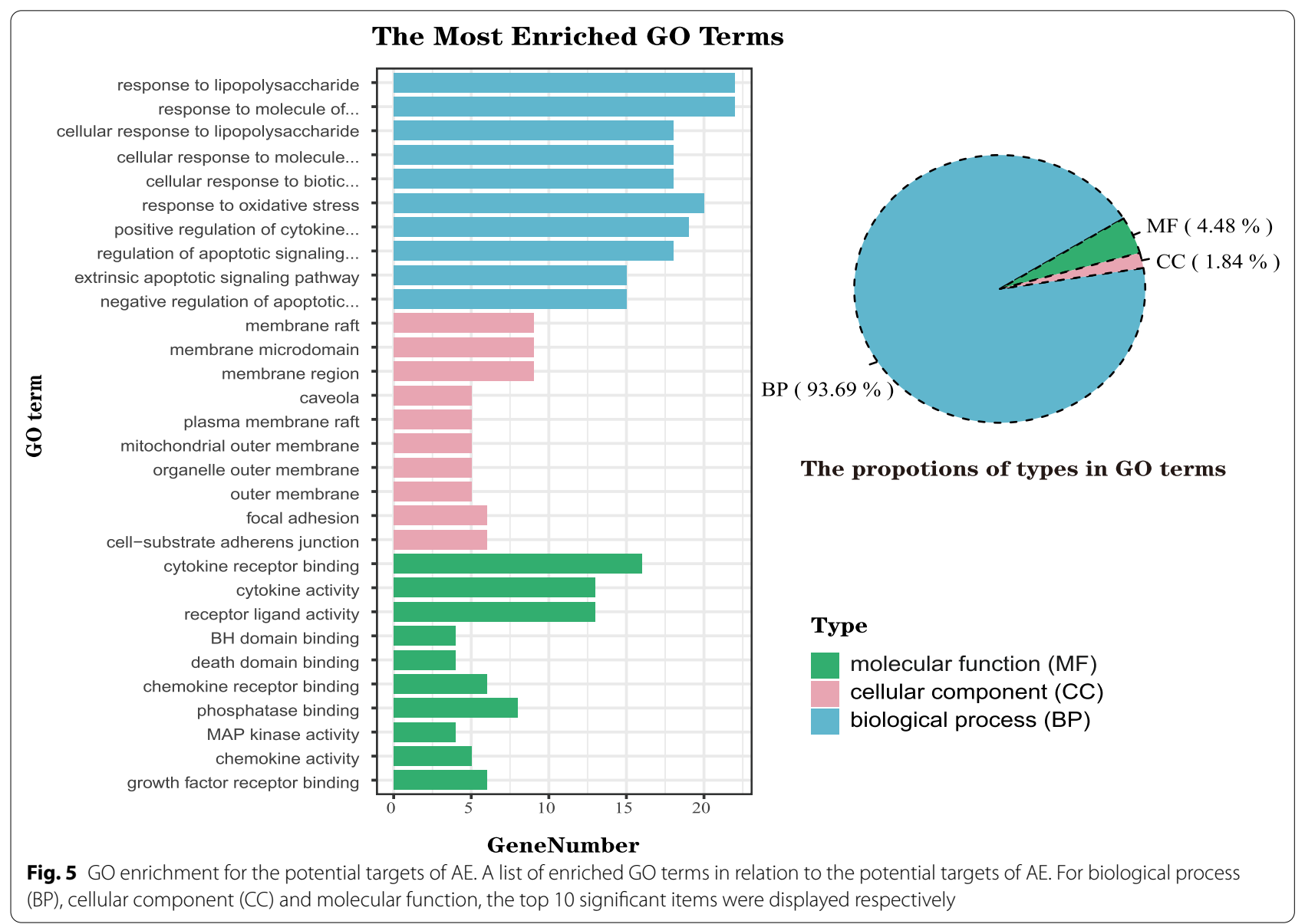

$\mathrm{ACV}$, have the characteristics of multiple compositions and interaction targets. The key ingredients in"AEcomponent-target-COVID" network were quercetin, kaempferol and luteolin. According to previous studies, quercetin has multiple biological activities, such as anti-inflammatory, anti-viral, anti-oxidative, antitumor actions [23, 24]. A study reported that quercetin could interact with the HA2 subunit of Influenza A virus (IAVs), and it could inhibit the entry of the H5N1 virus using the pseudovirus-based drug screening system [25]. Bacterial LPS activates the translocation of NF- $K B$ by binding to the TLR on the surface of the cell membrane, thereby triggering the pro-inflammatory responses, and quercetin can block the activation of TLR and inhibit the expression of LPS-induced adhesion molecules and inflammatory factors [26]. In addition, kaempferol has anti-inflammatory, anti-oxidative, anti-bacterial, anti-viral and other effects [27], including the inhibition of the replication of bovine herpes virus and LPS-induced inflammatory responses [28]. It could also alleviate acute lung injury induced by H9N2 swine influenza virus through inhibiting TLR4/myeloid differentiation factor 88 (MyD88)-mediated NF- $\kappa B$ and
MAPK signaling pathways [29]. On the other hand, luteolin has anti-inflammatory, anti-allergic, antibacterial, anti-viral and other effects [30]. For example, luteolin inhibits the replication of dengue virus by inhibiting pro-protein-converting enzyme activity [31]. It also has potent anti-viral activity against Japanese encephalitis virus replication in non-small-cell lung carcinoma A549 cells [32]. In conclusion, these suggested that the active components of $\mathrm{AE}$ can be used to treat many diseases with multi-target regulation, especially for viral infectious diseases.

According to the literature research, angiotensin-converting enzyme 2 (ACE2) and 3 C-like protease (3CL pro) are regarded as the critical targets for anti-viral drug design [33, 34]. TCM could target ACE2 to prevent SARS-CoV-2 from entering into the host cells or target $3 \mathrm{CL}$ pro to inhibit the replication and assembly of the virus in the cells. For examples, Li et al. found that Lianhua Qinwen capsule significantly inhibited the replication of SARS-CoV-2 with an $\mathrm{IC}_{50}$ value of $411.2 \mu \mathrm{g} / \mathrm{mL}$ in Vero E6 cells [10]. Fan et al. reported that the active ingredients of Qingfei Paidu decoction might act directly on the SARS-CoV-2 3CL pro to block viral proliferation [35]. 
a

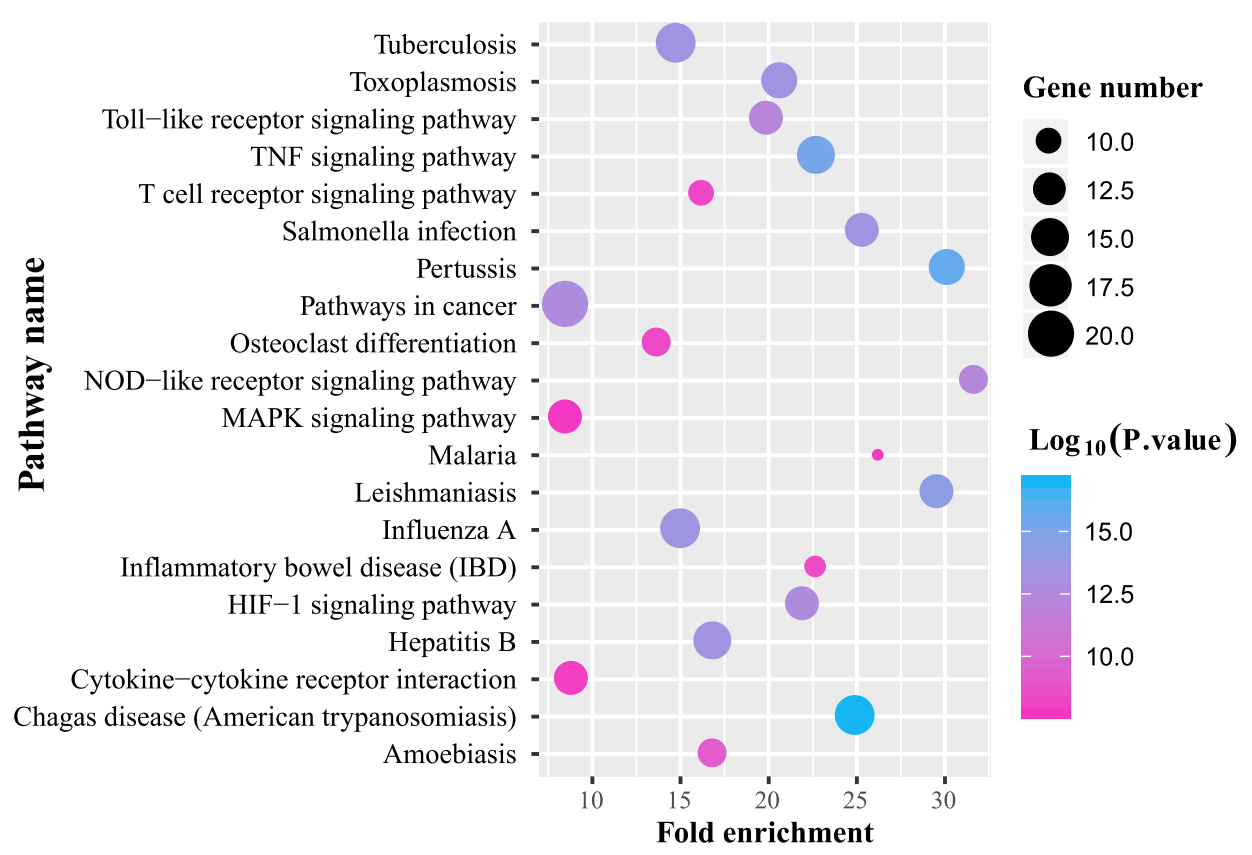

b

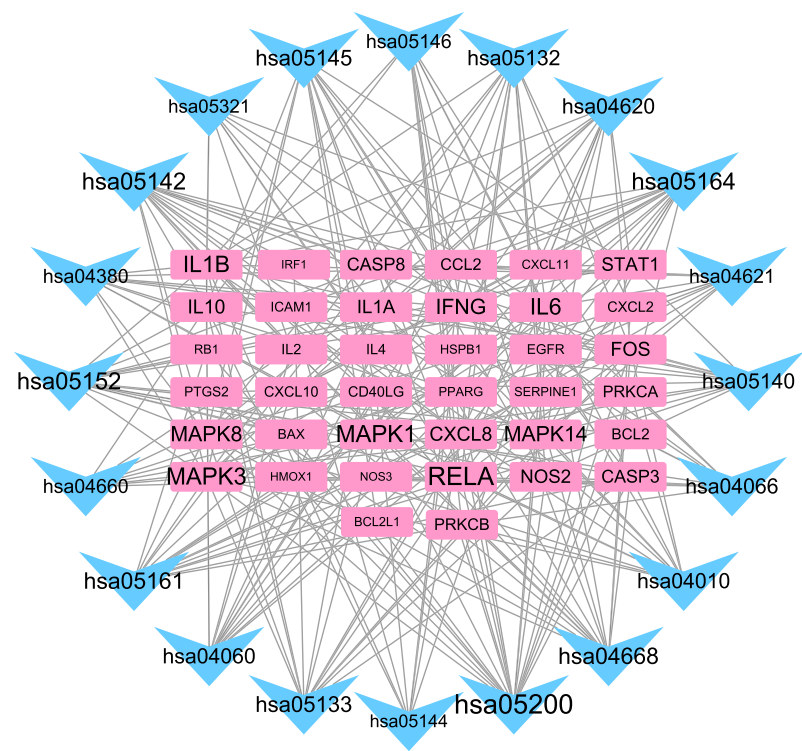

Fig. 6 The top 20 significant KEGG pathway analysis of shared targets between AE and COVID-19. a The KEGG pathway enrichment. The X-axis showed the enrichment scores of the pathways, while the Y-axis showed enriched KEGG categories of the targets. The size of the dots represents the counts of the genes, and the colour of the dots represents the $p$-value. $\mathbf{b}$ The top 20 significant pathways and target networks. Blue colour represents the pathway ID and red colour represents the potential targets. The larger the font, the greater the degree value of the nodes in the network

Besides, the virus can induce cell damage and induce a series of immune and inflammatory responses after entering into the cells. Especially in severe and critical patients, cytokine storms are closely related to the progression of the disease. In this study, 44 potential targets were obtained through the intersection of the disease targets of COVID-19 and the action targets of AE, which are mainly related to inflammatory or immune factors, such as IL-6, MAPK1, MAPK8, IL1B, RELA, CXCL-8, CCL2, and PTGS2. Furthermore, ESS and ACV might have 
Table 4 Target distribution information of the top 20 enriched KEGG pathways

\begin{tabular}{|c|c|c|}
\hline Terms & Description & Genes \\
\hline hsa05152 & Tuberculosis & $\begin{array}{l}\text { IL6, RELA, STAT1, IL10, MAPK1, CASP3, BAX, MAPK14, BCL2, CASP8, IFNG, MAPK3, IL1B, MAPK8, NOS2, } \\
\text { IL1A }\end{array}$ \\
\hline hsa05145 & Toxoplasmosis & $\begin{array}{l}\text { RELA, BCL2L1, STAT1, IL10, MAPK1, CASP3, CD40LG, MAPK14, BCL2, CASP8, IFNG, MAPK3, MAPK8, } \\
\text { NOS2 }\end{array}$ \\
\hline hsa04620 & Toll-like receptor signaling pathway & IL6, RELA, CXCL8, STAT1, CXCL11, CXCL10, MAPK1, FOS, MAPK14, CASP8, MAPK3, IL1B, MAPK8 \\
\hline hsa04668 & TNF signaling pathway & $\begin{array}{l}\text { ICAM1, IL6, CCL2, PTGS2, RELA, CXCL2, CXCL10, MAPK1, FOS, CASP3, MAPK14, CASP8, MAPK3, IL1B, } \\
\text { MAPK8 }\end{array}$ \\
\hline hsa04660 & T cell receptor signaling pathway & IL4, MAPK1, FOS, CD40LG, MAPK14, RELA, MAPK3, IFNG, IL10, IL2 \\
\hline hsa05132 & Salmonella infection & IL6, RELA, CXCL2, CXCL8, MAPK1, FOS, MAPK14, IFNG, MAPK3, IL1B, MAPK8, NOS2, IL1A \\
\hline hsa05133 & Pertussis & IL6, RELA, CXCL8, IL10, MAPK1, FOS, CASP3, MAPK14, MAPK3, IRF1, IL1B, MAPK8, NOS2, IL1A \\
\hline hsa05200 & Pathways in cancer & $\begin{array}{l}\text { PRKCA, EGFR, IL6, PTGS2, RELA, PPARG, CXCL8, RB1, BCL2L1, STAT1, PRKCB, MAPK1, FOS, CASP3, BAX, } \\
\text { BCL2, CASP8, MAPK3, MAPK8, NOS2 }\end{array}$ \\
\hline hsa04380 & Osteoclast differentiation & MAPK1, FOS, MAPK14, RELA, MAPK3, PPARG, IFNG, IL1B, MAPK8, STAT1, IL1A \\
\hline hsa04621 & NOD-like receptor signaling pathway & MAPK1, IL6, CCL2, MAPK14, RELA, MAPK3, CASP8, CXCL2, CXCL8, IL1B, MAPK8 \\
\hline hsa04010 & MAPK signaling pathway & PRKCA, EGFR, FOS, MAPK1, CASP3, MAPK14, RELA, MAPK3, HSPB1, IL1B, \\
\hline hsa05144 & Malaria & ICAM1, IL6, CCL2, CD40LG, IFNG, CXCL8, IL1B, IL10 \\
\hline hsa05140 & Leishmaniasis & IL4, PTGS2, RELA, STAT1, IL10, FOS, MAPK1, MAPK14, IFNG, MAPK3, IL1B, NOS2, IL1A \\
\hline hsa05164 & Influenza A & $\begin{array}{l}\text { PRKCA, ICAM1, IL6, CCL2, RELA, CXCL8, STAT1, PRKCB, CXCL10, MAPK1, MAPK14, IFNG, MAPK3, IL1B, } \\
\text { MAPK8, IL1A }\end{array}$ \\
\hline hsa05321 & Inflammatory bowel disease (IBD) & IL4, IL6, RELA, IFNG, IL1B, STAT1, IL10, IL1A, IL2 \\
\hline hsa04066 & HIF-1 signaling pathway & PRKCA, EGFR, MAPK1, IL6, RELA, HMOX1, BCL2, MAPK3, SERPINE1, IFNG, NOS3, NOS2, PRKCB \\
\hline hsa05161 & Hepatitis B & PRKCA, FOS, MAPK1, CASP3, IL6, BCL2, RELA, BAX, CASP8, MAPK3, CXCL8, MAPK8, RB1, STAT1, PRKCB \\
\hline hsa04060 & Cytokine-cytokine receptor interaction & IL4, IL6, CCL2, CXCL2, CXCL8, CXCL11, IL10, CXCL10, CD40LG, IFNG, IL1B, IL1A, IL2 \\
\hline hsa05142 & $\begin{array}{l}\text { Chagas disease } \\
\text { (American trypanosomiasis) }\end{array}$ & $\begin{array}{l}\text { IL6, CCL2, RELA, CXCL8, IL10, MAPK1, FOS, MAPK14, CASP8, IFNG, SERPINE1, MAPK3, IL1B, MAPK8, } \\
\text { NOS2, IL2 }\end{array}$ \\
\hline hsa05146 & Amoebiasis & PRKCA, CASP3, IL6, RELA, IFNG, CXCL8, HSPB1, IL1B, NOS2, IL10, PRKCB \\
\hline
\end{tabular}

Table 5 Binding energy values of compounds in AE with SARS-CoV-2 3CL pro and ACE2

\begin{tabular}{|c|c|c|c|c|c|}
\hline \multirow[t]{2}{*}{ Active components } & \multirow[t]{2}{*}{ Formula } & \multirow[t]{2}{*}{ Molecular weight } & \multirow[t]{2}{*}{ CAS number } & \multicolumn{2}{|c|}{ Binding energy $(\mathrm{kJ} / \mathrm{mol})$} \\
\hline & & & & 3CLpro & ACE2 \\
\hline Quercetin & $\mathrm{C} 15 \mathrm{H} 1007$ & 302.25 & $117-39-5$ & $-30.1 \pm 0.6$ & $-34.3 \pm 0.0$ \\
\hline Kaempferol & $\mathrm{C} 15 \mathrm{H} 1006$ & 286.25 & $520-18-3$ & $-29.5 \pm 1.4$ & $-33.4 \pm 0.6$ \\
\hline Luteolin & $\mathrm{C} 15 \mathrm{H} 1006$ & 286.25 & $491-70-3$ & $-29.7 \pm 1.6$ & $-34.3 \pm 0.0$ \\
\hline beta-Sitosterol & $\mathrm{C} 29 \mathrm{H} 50 \mathrm{O}$ & 414.69 & $83-46-5$ & $-28.1 \pm 0.2$ & $-36.5 \pm 1.6$ \\
\hline Naringenin & $\mathrm{C} 15 \mathrm{H} 12 \mathrm{O} 5$ & 272.25 & $480-41-1$ & $-30.1 \pm 0.6$ & $-33.0 \pm 0.0$ \\
\hline Stigmasterol & $\mathrm{C} 29 \mathrm{H} 48 \mathrm{O}$ & 412.7 & $83-48-7$ & $-30.8 \pm 1.9$ & $-36.5 \pm 1.5$ \\
\hline I-SPD & $\mathrm{C} 19 \mathrm{H} 21 \mathrm{NO} 4$ & 327.4 & $6562-13-3$ & $-28.3 \pm 0.7$ & $-34.7 \pm 0.0$ \\
\hline Estrone & $\mathrm{C} 18 \mathrm{H} 22 \mathrm{O} 2$ & 270.4 & 53-16-7 & $-29.8 \pm 0.2$ & $-33.4 \pm 0.0$ \\
\hline Glabridin & $\mathrm{C} 2 \mathrm{OH} 20 \mathrm{O} 4$ & 324.4 & $59870-68-7$ & $-30.0 \pm 2.8$ & $-35.9 \pm 0.6$ \\
\hline Machiline & $\mathrm{C} 17 \mathrm{H} 19 \mathrm{NO} 3$ & 285.34 & $2196-60-3$ & $-25.6 \pm 0.7$ & $-30.9 \pm 0.3$ \\
\hline
\end{tabular}

synergistic effects, as they do not only have common action targets, but also different action targets, which could be explained by the combined actions of the two herbs from the molecular level. From the PPI network, IL-6 was found to be the most critical target. IL-6 is a powerful inducer of the acute-phase reaction, which can act on $\mathrm{B}$ and $\mathrm{T}$ cells, liver cells, hematopoietic progenitor cells and central nervous system cells, and can induce multiple biological processes [36]. A recent study showed that $\mathrm{CD} 4^{+} \mathrm{T}$ cells were rapidly activated in response to SARS-COV-2 infection, and they were then differentiated into Th1 cells that can produce granulocyte-macrophage 


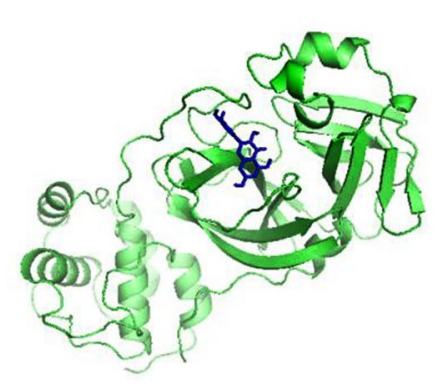

Quercetin+3CLpro

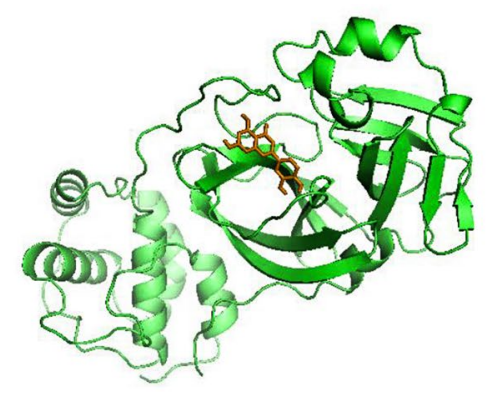

Luteolin+3CLpro

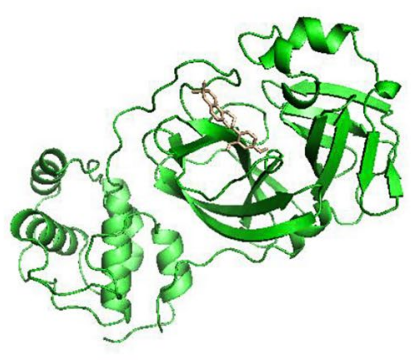

Glabridin+3CLpro

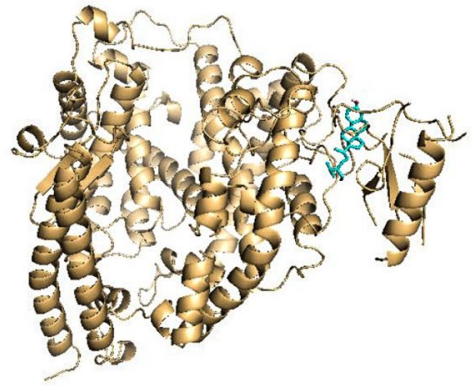

Beta-sitosterol+ACE2

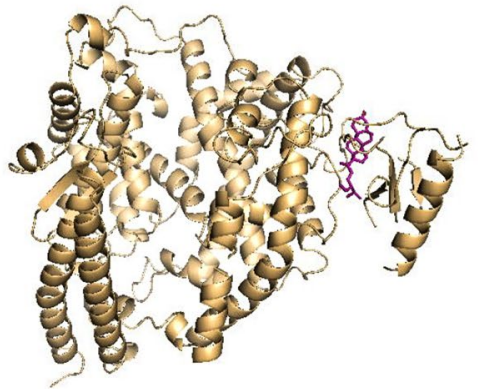

Stigmasterol + ACE2

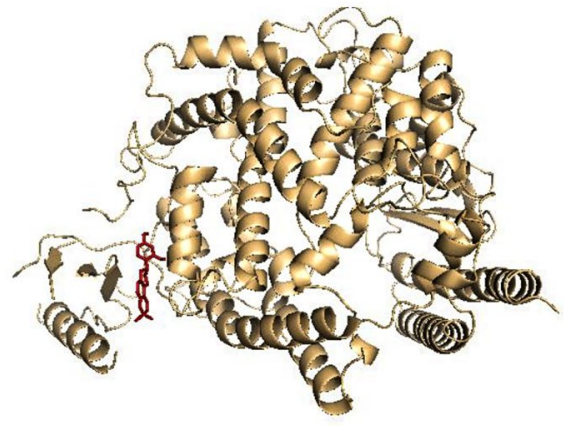

Glabridin + ACE2

Fig. 7 Molecular docking diagram of SARS-CoV 3CL hydrolase and ACE2 with core compounds of AE

colony stimulating factor (GM-CSF). GM-CSF further activated inflammatory $\mathrm{CD} 14^{+} \mathrm{CD} 16^{+}$monocytes to secrete IL-6 and other factors to amplify inflammation. Therefore, we speculated that GM-CSF and IL- 6 may be the key factors to induce cytokine storm [37]. In addition, GO enrichment analysis revealed that the biological processes of AE against COVID-19 involved cytokine activity, anti-oxidative stress response, cell apoptosis and cellular response to LPS and bacteria-derived molecules. The molecular functions of AE mainly included cytokine receptor binding, cytokine and receptor ligand activities, chemokine receptor binding, phosphatase binding, MAP kinase activity, and chemokine activity. From the KEGG enrichment analysis, our results indicated that the key signaling pathways of AE against COVID-19 are TNF, TLR, HIF-1 and NLRs, which are closely related to inflammation, immunity and oxidation process. Similarly, many studies also reported that the anti-COVID-19 mechanisms of TCM prescriptions with AE might be related to these above pathways [38-40]. In particular, TNF pathway has been reported to be involved in a variety of physiological and pathological processes, such as cell proliferation, apoptosis, and modulation of immune responses and induction of inflammation [41, 42], so it may play an important role in the treatment of COVID19. Moreover, the molecular docking results showed that the main active compounds in AE combine with SARSCOV-2 3CL pro and ACE2 with a lower binding energy, which also suggests that $\mathrm{AE}$ with multiple components have a potential therapeutic effect on COVID-19.

In summary, this study innovatively employed the association rule approach to examine the distribution and combination compatibility of TCM recommended by leading experts for the treatment of COVID-19. This allows us to better grasp the clinical use of TCM treatment. Besides, the combination of ACV and ESS was selected as the most important herbal pair for the treatment of COVID-19. AE may has therapeutic effects against COVID-19 by affecting the pathological processes such as inflammatory and immune responses, cell apoptosis, hypoxia damage and other pathological processes through multiple components, targets and pathways. The molecular docking results also suggests that $\mathrm{AE}$ with multiple components have a potential 
therapeutic effect on COVID-19. However, it should be noted that there is still lack of cognition on the pathogenesis of COVID-19 now, and different methods of computational chemistry and biology have different results and limitations, so it could be possible that the calculated results are not consistent with the actual therapeutic effects. Therefore, it is necessary to further conduct in vitro and in vivo experiments to confirm the efficacy of AE for the treatment of COVID-19, thus providing more information for its development and clinical application.

\section{Conclusions}

In conclusion, the combination of ACV and ESS was identified to be the most important herbal pair for the treatment of COVID-19. The main active ingredients of $\mathrm{AE}$ against COVID-19 were quercetin, kaempferol and luteolin, and the important targets were IL-6, MAPK1, MAPK8, IL-1 $\beta$, and RELA. The results suggested that AE might have therapeutic effects against COVID-19 by affecting the inflammatory and immune responses, cell apoptosis, hypoxia damage and other pathological processes through multiple components, targets and pathways.

\begin{abstract}
Abbreviations
ACE2: Angiotensin-converting enzyme 2; ACV: Amygdalus Communis Vas; ADME: Absorption, distribution, metabolism, excretion; BP: Biological process; CC: Cellular component; CCL: C-C motif chemokine ligand; COVID-19: Coronavirus disease 2019; CXCL: C-X-C motif chemokine ligand; DL: Drug-likeness, ESS: Ephedra sinica Stapf; GM-CSF: Granulocyte-macrophage colony stimulating factor; GO: Gene Ontology; H1 N1: Influenza A; HIF-1: Hypoxia-inducible factor-1; IAVs: Influenza A virus; IL-6: Interleukin 6; KEGG: Kyoto Encyclopedia of Genes and Genomes; LPS: Lipopolysaccharide; MAPK: Mitogen-activated protein kinase; MF: Molecular function; NF-kB: Nuclear factor kappa-lightchain-enhancer of activated B cells; NOD: Nucleotide-binding oligomerization domain; OB: Oral bio-availability; OMIM: Online mendelian inheritance in man; PPI: Protein-protein interaction; PTGS: Prostaglandin G/H synthase; RELA: Nuclear factor kappa-light-chain-enhancer of activated B cells p65 subunit; SARS: Severe acute respiratory syndrome; TCM: Traditional Chinese medicine; TCMSP: Traditional Chinese medicine systems pharmacology database; TLR: Toll-like receptor; TNF: Tumor necrosis factor.
\end{abstract}

\section{Acknowledgements}

We would like to thank Professor Yitao Wang from University of Macau for the suggestions on the research methods of this study.

\section{Authors' contributions}

Conceptualization, SX and CL; Methodology, SX, JC and BG; Software, SX and HG.; Validation, SX, ZZ, BG, CTV, XL, JC, HG, GC and CL; Formal Analysis, SC and XL; Investigation, JC and ZZ; Resources, CL; Data Curation, CTV and HG; Writing-Original Draft Preparation, SX; Writing-Review \& Editing, ZZ and CTV; Visualization, SX and BG; Supervision, GC and CL; Project Administration, $\mathrm{CL}$; Funding Acquisition, $\mathrm{CL}$ and GC. All authors read and approved the final manuscript.

\section{Funding}

This work was supported by the Joint Fund Project of National Natural Science Foundation of China (U1705286) and the Macao Science and Technology Development Fund (014/2017/A1).

\section{Availability of data and materials}

The data used to support the findings of this study are available from the corresponding authors upon request.

\section{Ethics approval and consent to participate}

Not applicable.

\section{Consent for publication}

The manuscript is approved by all the authors for publication.

\section{Competing interests}

The authors report that there are no conflicts of interest in this work.

\section{Author details}

${ }^{1}$ Research Base of Traditional Chinese Medicine Syndrome, Fujian University of Traditional Chinese Medicine, No.1 Qiuyang Road, Minhou District, 350122 Fuzhou, China. ${ }^{2}$ State Key Laboratory of Quality Research in Chinese Medicine, Institute of Chinese Medical Sciences, University of Macau, 999078 Macao, China. ${ }^{3}$ Department of Internal Medicine, The Third People's Hospital, Fujian University of Traditional Chinese Medicine, 350108 Fuzhou, China.

Received: 22 July 2020 Accepted: 22 January 2021

Published online: 03 March 2021

\section{References}

1. Kandel N, Chungong S, Omaar A, et al. Health security capacities in the context of COVID-19 outbreak: an analysis of International Health Regulations annual report data from 182 countries. Lancet. 2020;395(10229):1047-53. https://doi.org/10.1016/S0140-6736(20)30553 $-5$.

2. Guan WJ, Ni ZY, Hu Y, et al. Clinical characteristics of coronavirus disease 2019\&nbsp;in China. N Engl J Med. 2020;382(18):1708-20. https://doi. org/10.1056/NEJMoa2002032

3. Rothe C, Schunk M, Sothmann P, et al. Transmission of 2019-nCoV infection from an asymptomatic contact in Germany. N Engl J Med. 2020;382(10):970-1. https://doi.org/10.1056/NEJMc2001468.

4. Zhao H, Lu X, Deng Y, et al. COVID-19: asymptomatic carrier transmission is an underestimated problem. Epidemiol Infect. 2020;148:e116. doi:https ://doi.org/10.1017/s0950268820001235.

5. Jin $\mathrm{YH}$, Cai L, Cheng ZS, et al. A rapid advice guideline for the diagnosis and treatment of 2019 novel coronavirus (2019-nCoV) infected pneumonia (standard version). Mil Med Res. 2020;7(1):4. https://doi.org/10.1186/ s40779-020-0233-6.

6. Cao HX. In response to the emergence of infectious diseases, traditional Chinese medicine should do something. China News of Traditional Chinese Medicine; 2013. https://kns.cnki.net/kcms/detail/detail.aspx?FileN ame $=$ ZYYB201308140030\&DbName $=C C N D 2013$.

7. Liu M, Gao Y, Yuan Y, et al. Efficacy and safety of integrated traditional chinese and western medicine for corona virus disease 2019 (COVID-19): a systematic review and meta-analysis. Pharmacol Res. 2020;158:104896. https://doi.org/10.1016/j.phrs.2020.104896.

8. General Office of National Health Commission of the People's Republic of ChinaOffice of National Administration of Traditional Chinese Medicine. Diagnosis and treatment of corona virus disease-19 (7 edition). China Med. 2020;15(06):801-5. https://doi.org/10.3760/j. issn.1673-4777.2020.06.001.

9. Runfeng $\mathrm{L}$, Yunlong $\mathrm{H}$, Jicheng $\mathrm{H}$, et al. Lianhuaqingwen exerts antiviral and anti-inflammatory activity against novel coronavirus (SARSCoV-2). Pharmacol Res. 2020;156:104761. doi:https://doi.org/10.1016/j. phrs.2020.104761.

10. Chen J, Wang YK, Gao Y, et al. Protection against COVID-19 injury by qingfei paidu decoction via anti-viral, anti-inflammatory activity and metabolic programming. Biomed Pharmacother. 2020;129:1 10281. doi:https://doi.org/10.1016/j.biopha.2020.110281.

11. Xia SJ, Chen SJ, Wu CW, et al. Novel coronavirus pneumonia diagnosis and treatment by "five differentiation " thinking. Tianjin J Tradit Chin Med. 2020;37(07):726-32. 
12. You $X, X u Y$, Huang J, et al. A data mining-based analysis of medication rules in treating bone marrow suppression by kidney-tonifying method. Evid Based Complement Alternat Med. 2019;2019:1907848. https://doi. org/10.1155/2019/1907848.

13. Li S, Zhang B, Jiang D, et al. Herb network construction and co-module analysis for uncovering the combination rule of traditional Chinese herbal formulae. BMC Bioinformatics. 2010;11(Suppl 11):6. https://doi. org/10.1186/1471-2105-11-S11-S6.

14. Cheng $Y$, Xiong $Y$. Research and improvement of apriori algorithm for association rules. Phys Rev A. 2016. https://doi.org/10.1109/IWISA 2010.5473473.

15. Ru J, Li P, Wang J, et al. TCMSP: a database of systems pharmacology for drug discovery from herbal medicines. J Cheminform. 2014;6:13. https:// doi.org/10.1186/1758-2946-6-13.

16. Barton HA, Pastoor TP, Baetcke K, et al. The acquisition and application of absorption, distribution, metabolism, and excretion (adme) data in agricultural chemical safety assessments. Crit Rev Toxicol. 2008;36(1):9-35. https://doi.org/10.1080/10408440500534362.

17. Rebhan M, Chalifa-Caspi V, Prilusky J, et al. GeneCards: a novel functional genomics compendium with automated data mining and query reformulation support. Bioinformatics. 1998;14(8):656-64. https://doi. org/10.1093/bioinformatics/14.8.656.

18. Hamosh A, Scott AF, Amberger JS, et al. Online Mendelian Inheritance in Man (OMIM), a knowledgebase of human genes and genetic disorders. Nucleic Acids Res. 2005;33(Database issue):D514-7. doi:https://doi. org/10.1093/nar/gki033.

19. Szklarczyk D, Morris JH, Cook H, et al. The STRING database in 2017: quality-controlled protein-protein association networks, made broadly accessible. Nucleic Acids Res. 2017;45(D1):D362-8. doi:https://doi. org/10.1093/nar/gkw937.

20. Tan A, Huang $H$, Zhang $P$, et al. Network-based cancer precision medicine: a new emerging paradigm. Cancer Lett. 2019;458:39-45. https://doi. org/10.1016/j.canlet.2019.05.015.

21. Hahsler M, Grün B, Hornik K. Introduction to arules-A computational environment for mining association rules and frequent item sets. Compr R Arch Netw. 2009. https://doi.org/10.18637/jss.v014.i15.

22. Wang Q, Gu XH, Liu QQ. COVID-19 Chinese medicine diagnosis and treatment manual. Beijing: China Press of Traditional Chinese Medicine; 2020

23. Rather RA, Bhagat M. Quercetin as an innovative therapeutic tool for cancer chemoprevention: molecular mechanisms and implications in human health. Cancer Med. 2019. https://doi.org/10.1002/cam4.1411.

24. Dabeek WM, Marra MV. Dietary quercetin and kaempferol: bioavailability and potential cardiovascular-related bioactivity in humans. Nutrients. 2019;11(10):2288. https://doi.org/10.3390/nu11102288.

25. Wu W, Li R, Li X, et al. Quercetin as an antiviral agent Inhibits Influenza A virus (IAV) entry. Viruses. 2015;8(1):6. https://doi.org/10.3390/v8010006.

26. Byun EB, Yang MS, Choi HG, et al. Quercetin negatively regulates TLR4 signaling induced by lipopolysaccharide through Tollip expression. Biochem Biophys Res Commun. 2013;431(4):698-705. doi:https://doi. org/10.1016/j.bbrc.2013.01.056.

27. Chen AY, Chen YC. A review of the dietary flavonoid, kaempferol on human health and cancer chemoprevention. Food Chem. 2013;138(4):2099-107. doi:https://doi.org/10.1016/j.foodc hem.2012.11.139.

28. Zhu L, Wang P, Yuan W, et al. Kaempferol inhibited bovine herpesvirus 1 replication and LPS-induced inflammatory response. Acta Virol. 2018;62(2):220-5. doi:https://doi.org/10.4149/av_2018_206.
29. Zhang R, Ai X, Duan Y, et al. Kaempferol ameliorates H9N2 swine influenza virus-induced acute lung injury by inactivation of TLR4/MyD88-mediated NF-kappaB and MAPK signaling pathways. Biomed Pharmacother. 2017;89:660-72. doi:https://doi.org/10.1016/j.biopha.2017.02.081.

30. Manzoor MF, Ahmad N, Ahmed Z, et al. Novel extraction techniques and pharmaceutical activities of luteolin and its derivatives. J Food Biochem. 2019;43(9):e12974. doi:https://doi.org/10.1111/jfbc.12974.

31. Peng $M$, Watanabe $S$, Chan KWK, et al. Luteolin restricts dengue virus replication through inhibition of the proprotein convertase furin. Antiviral Res. 2017;143:176-85. doi:https://doi.org/10.1016/j.antiviral.2017.03.026.

32. Fan W, Qian S, Qian P, et al. Antiviral activity of luteolin against Japanese encephalitis virus. Virus Res. 2016;220:112-6. doi:https://doi.org/10.1016/j. virusres.2016.04.021.

33. Zhang H, Penninger JM, Li Y, et al. Angiotensin-converting enzyme 2 (ACE2) as a SARS-CoV-2 receptor: molecular mechanisms and potential therapeutic target. Intensive care medicine. 2020;46(4):586-90. doi:https ://doi.org/10.1007/s00134-020-05985-9.

34. Jo S, Kim S, Shin DH, et al. Inhibition of SARS-CoV 3CL protease by flavonoids. J Enzyme Inhib Med Chem. 2020;35(1):145-51. doi:https://doi. org/10.1080/14756366.2019.1690480.

35. Fan J, Qin X, Li Z. Mechanism of Farfarae Flosin Qingfei Paidu Decoction against COVID-19 based on network pharmacology and molecular docking. Chinese Traditional Herbal Drugs. 2020;51(09):2317-25.

36. Kang S, Tanaka T, Narazaki M, et al. Targeting interleukin-6 signaling in clinic. Immunity. 2019;50(4):1007-23. doi:https://doi.org/10.1016/j.immun i.2019.03.026

37. Zhou Y, Fu B, Zheng $X$, et al. Pathogenic T-cells and inflammatory monocytes incite inflammatory storms in severe COVID-19 patients. Nat SCi Rev. 2020;7:998-1002. https://doi.org/10.1093/nsr/nwaa041.

38. Huang YF, Bai C, He F, et al. Review on the potential action mechanisms of Chinese medicines in treating Coronavirus Disease 2019 (COVID-19). Pharmacol Res. 2020;158:104939. https://doi.org/10.1016/j. phrs.2020.104939.

39. Luo E, Zhang D, Luo H, et al. Treatment efficacy analysis of traditional Chinese medicine for novel coronavirus pneumonia (COVID-19): an empirical study from Wuhan, Hubei Province, China. Chin Med. 2020;15:34. doi:https://doi.org/10.1186/s13020-020-00317-x.

40. Shi-Ying Z, Ling L, Ning Z, et al. Systematic pharmacological strategies to explore the regulatory mechanism of Ma Xing Shi Gan decoction on COVID-19. Digital Chin Med. 2020;3(2):96-115. https://doi.org/10.1016/j. dcmed.2020.06.004

41. Thoh M, Kumar P, Nagarajaram HA, et al. Azadirachtin interacts with the tumor necrosis factor (TNF) binding domain of its receptors and inhibits TNF-induced biological responses. J Biol Chem. 2010;285(8):5888-95. doi:https://doi.org/10.1074/jbc.M109.065847.

42. Coondoo A. Cytokines in dermatology - a basic overview. Indian J Dermatol. 2011;56(4):368-74. https://doi.org/10.4103/0019-5154.84717.

\section{Publisher's note}

Springer Nature remains neutral with regard to jurisdictional claims in published maps and institutional affiliations.

Ready to submit your research? Choose BMC and benefit from

- fast, convenient online submission

- thorough peer review by experienced researchers in your field

- rapid publication on acceptance

- support for research data, including large and complex data types

- gold Open Access which fosters wider collaboration and increased citations

- maximum visibility for your research: over 100M website views per year

At BMC, research is always in progress.

Learn more biomedcentral.com/submissions 